\title{
Processing of Interstellar Dust Grains in Galaxies Revealed by AKARI
}

\author{
Hidehiro Kaneda $^{1}$, Daisuke Ishihara ${ }^{1}$, Koudai Kobata ${ }^{1}$, Toru Kondo ${ }^{1}$, \\ Mitsuyoshi Yamagishi ${ }^{1}$, Akiko Yasuda ${ }^{1}$, Takashi Onaka ${ }^{2}$, Itsuki \\ Sakon $^{2}$, and Toyoaki Suzuki ${ }^{3}$ \\ ${ }^{1}$ Graduate School of Science, Nagoya University, Chikusa-ku, Nagoya, 464-8602, Japan \\ email: kaneda@u.phys.nagoya-u.ac.jp \\ ${ }^{2}$ Department of Astronomy, Graduate School of Science, University of Tokyo, Bunkyo-ku, \\ Tokyo 113-0033, Japan \\ ${ }^{3}$ Institute of Space and Astronautical Science, Japan Aerospace Exploration Agency, \\ Sagamihara, Kanagawa 252-5210, Japan
}

\begin{abstract}
With AKARI, we have performed a systematic study of interstellar dust grains in various environments of galaxies. In many cases, the IR emission of dust is an important tool to trace star-forming activities in galaxies. However it is much more than just star-formation tracers. AKARI has revealed the detailed properties of dust grains in regions not relevant to star formation as well, some of which are found not to follow our old empirical knowledge. Because of its unique capabilities, such as near- and far-IR spectroscopy, and all-sky coverage, AKARI has provided new knowledge on the processing of carbonaceous grains including polycyclic aromatic hydrocarbons. We present the latest results obtained from our AKARI observations of the ISM in our Galaxy and nearby galaxies.
\end{abstract}

Keywords. dust, extinction — galaxies: ISM — Galaxy: center

\section{Introduction}

In star-forming regions, dust and polycyclic aromatic hydrocarbons (PAHs) absorb a significant fraction of stellar ultraviolet photons and re-radiate them in the infrared (IR). Hence the IR luminosities due to dust and PAH emission are both powerful tools to trace star-forming activities in galaxies. Beyond such star-formation tracers, however, spectral information on the dust and $\mathrm{PAH}$ emission would have much deeper physical implications for understanding the properties of the ISM.

We have performed a systematic study of interstellar dust grains in various environments of galaxies including our Galaxy, within the framework of the AKARI mission program, "ISM in our Galaxy and nearby galaxies" (Kaneda et al. 2009). AKARI has revealed the detailed properties of dust grains in regions not relevant to the star formation as well, some of which are found not to follow our old empirical knowledge. Because of its unique capabilities, such as near- and far-IR spectroscopy, and all-sky coverage in the mid- and far-IR, AKARI has provided new knowledge on the processing of dust, particularly carbonaceous grains including polycyclic aromatic hydrocarbons (PAHs), in the interstellar space.

The primary purpose of the AKARI mission was to conduct all-sky surveys at six photometric bands (9, 18, 65, 90, 140, and $160 \mu \mathrm{m}$; Murakami et al. 2007). In particular, the $9 \mu \mathrm{m}$ diffuse map is the first all-sky map of the PAH emission. The top panel in Fig. 1 shows the Galactic plane $9 \mu \mathrm{m}$ map of an area of about $175^{\circ} \times 60^{\circ}\left(l=-80^{\circ} \sim+95^{\circ}\right.$, $|b|<30^{\circ}$ ), which is created from the all-sky survey data with the Infrared Camera (IRC; 
Onaka et al. 2007), shown together with examples of near-IR spectra taken with the IRC. The bottom panel shows the $90 \mu \mathrm{m}$ map from the Far-Infrared Surveyor (FIS; Kawada et al. 2007) all-sky survey of the same area as the $9 \mu \mathrm{m}$ map, together with examples of the far-IR spectra taken with the FIS. The positions of spectroscopic observations with the IRC and the FIS are plotted on the $9 \mu \mathrm{m}$ and the $90 \mu \mathrm{m}$ map, respectively.

\section{ISM diagnostics: carbon dust chemistry}

Ishihara et al. (2011) derived the spatial distribution of carbon-rich AGB stars in our Galaxy using the AKARI mid-IR point source catalog (Ishihara et al. 2010). In general, carbonaceous grains are created and supplied to interstellar space through stellar massloss winds from carbon stars. By comparing their distribution with that of the PAH emission from the $9 \mu \mathrm{m}$ all-sky diffuse map (Fig. 1), we are investigating the relationships between the supplying sources and the resultant distributions (Ishihara et al. in prep.). It is known that large carbonaceous grains are mostly amorphous and partially hydrogenated in the interstellar space, but they are likely to be graphitized or crystallized somewhere because a significant amount of pre-solar carbonaceous grains are found to be graphite and (nano-)diamond. One problem is that very high annealing temperatures $(2000 \sim 3000 \mathrm{~K})$ would be needed to graphitize or crystallize carbonaceous grains. On the other hand, very small carbonaceous grains are relatively easily graphitized to PAHs, thanks to their small heat capacity, at phases of proto-planetary to planetary nebulae. PAHs are widely distributed in the Galactic plane, similarly to large grains, as revealed by the AKARI all-sky maps. Then PAHs may experience some structural
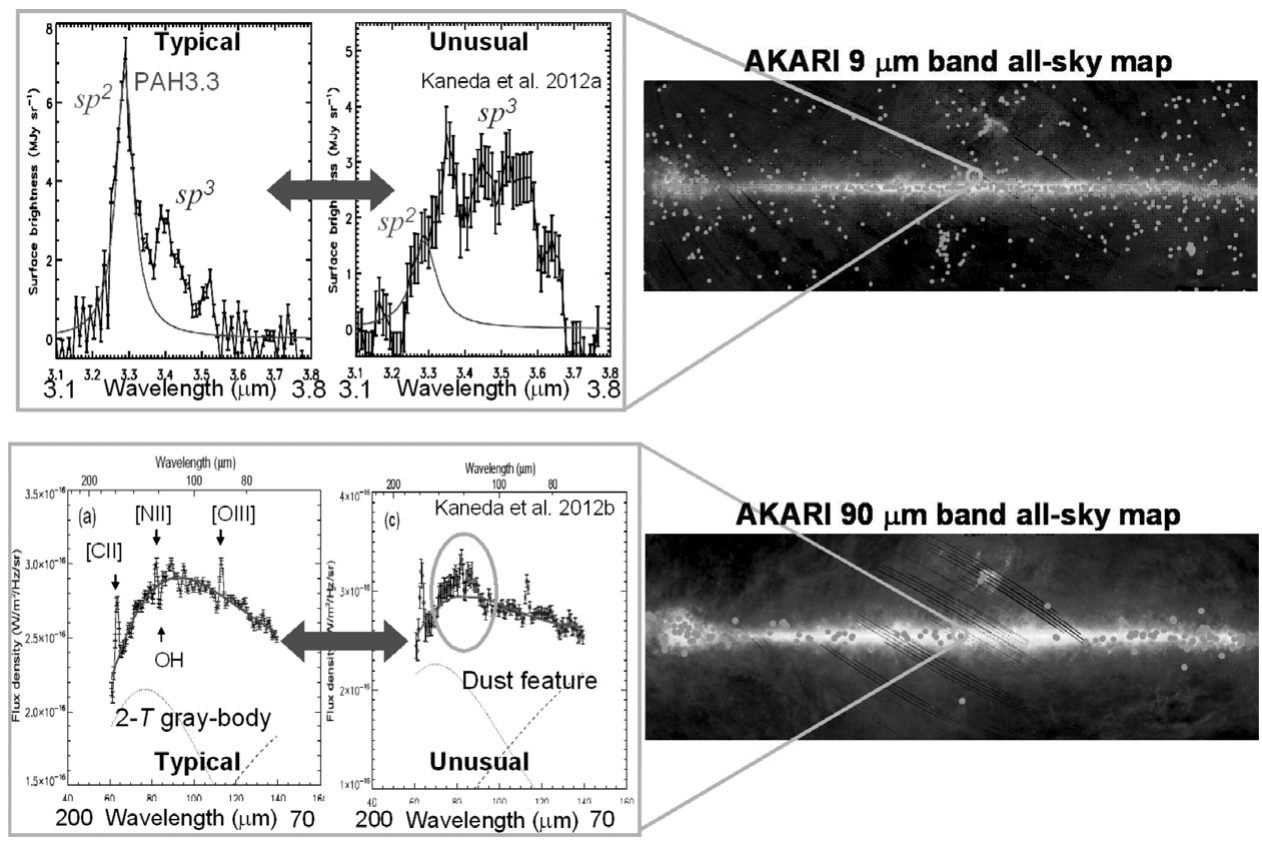

Figure 1. (Top) Galactic plane $9 \mu \mathrm{m}$ map for an area of about $175^{\circ} \times 60^{\circ}\left(l=-80^{\circ} \sim+95^{\circ}\right.$, $|b|<30^{\circ}$ ) created from the IRC all-sky survey data, where the positions of spectroscopic observations with the IRC are plotted. (Bottom) The $90 \mu \mathrm{m}$ map from the FIS all-sky survey for the same area as the $9 \mu \mathrm{m}$ map, with the positions of spectroscopic observations with the FIS. Examples of the near-IR (top) and far-IR (bottom) typical and unusual spectra taken with the IRC and FIS, respectively, are shown together. 
changes in harsh interstellar environments, which results in increasing aliphatic ratios in mixed aromatic-aliphatic hydrocarbon structures (Kwok et al. 2011). In dense molecular clouds, once such hydrocarbons are incorporated into icy grain mantles, they can be evolved into more complex organic compounds, potentially providing pre-biotic materials to planet-forming systems. In light of the evolution of carbonaceous dust, we below present our AKARI results on the processing of hydrocarbon particles and formation of graphite grains.

\section{Results}

Structural changes of hydrocarbons. The top panel in Fig. 1 shows the AKARI 3.1$3.8 \mu \mathrm{m}$ spectrum obtained for the foot points of CO molecular loops near the Galactic center (Kaneda et al. 2012a). The CO molecular loops were found by NANTEN; Fukui et al. (2006) suggested that the molecular loops were formed by the buoyant rise of magnetic loops due to magnetic flotation driven by the Parker instability. As compared with the typical spectrum of the diffuse ISM, which shows strong PAH emission at $3.3 \mu \mathrm{m}$ with relatively weak sub-features at 3.4-3.6 $\mu \mathrm{m}$, the $3.4-3.6 \mu \mathrm{m}$ features are unusually abundant at the foot points of the molecular loop, suggesting the dominance of aliphatic structures over aromatic ones. Our result calls for such a condition in the magnetic flotation picture that the falling speed of the gas along the loop should be fast enough to alter the properties of hydrocarbon grains by shocks at its foot points.

Similar results, i.e., notably strong aliphatic features, have so far been obtained for the galactic superwind regions in the halo of M 82 (Yamagishi et al. 2012) and the inner bar connecting the starburst ring and the central AGN in NGC 1097 (Kondo et al. 2012). As for M82, we find that copious amounts of large grains and PAHs are flowing out of the disk through galactic superwinds (Kaneda et al. 2010). Yamagishi et al. (2012) clearly detect the PAH $3.3 \mu \mathrm{m}$ emission and the 3.4-3.6 $\mu \mathrm{m}$ features in the halo regions, which are located at a distance of $2 \mathrm{kpc}$ away from the galactic center, thus confirming the presence of very small PAHs even in the harsh environment of the M82 halo. The 3.4-3.6 $\mu \mathrm{m}$ features are unusually abundant in the halo, suggesting shattering of carbonaceous grains in shocks. Kondo et al. (2012) detect strong aliphatic emission from the inner bar of NGC 1097, which indicates that the gas and dust in the bar are in turbulent motion, likely fueling the central AGN from the starburst ring.

Formation of graphite grains in the Galactic center. The bottom panel in Fig. 1 shows the AKARI 70-160 $\mu \mathrm{m}$ spectrum obtained for a cloud in the Galactic center. AKARI is capable of far-IR spectral mapping with the imaging FTS (FIS-FTS; Kawada et al. 2008). For the Galactic center, we investigated spatial variations in the spectral shapes, focusing on dust continuum emission (Kaneda et al. 2012b). For this purpose, we reduced the spectral resolution to increase the signal-to-noise ratio of the dust continuum emission. The spectral fitting shows that two modified blackbody components with temperatures of $\sim 20 \mathrm{~K}$ and $\sim 50 \mathrm{~K}$ can reproduce most of the continuum spectra. For some spectra, however, we find that there is a significant excess on top of the modified blackbody continuum around 110-130 $\mu \mathrm{m}$, as shown in the bottom panel of Fig. 1 . The excess emission probably represents a dust feature and possible origins of the dust feature were discussed in Kaneda et al. (2012b). We find that a dust model including large graphite grains can reproduce the observed spectrum with the dust feature fairly well. The detection of the excess feature from this region might be related to the (past) activity of the Galactic center, because thermal annealing with very high temperatures is needed to graphitize carbonaceous grains. This result may also be related to the abundant PAHs 
at the centers of elliptical galaxies (e.g. Kaneda et al. 2011), because many elliptical galaxies are known to possess galactic nuclei with declining or ceased activity like our Galaxy. It is also reported that some AGN dust tori show dust temperatures that would be too high to be explained by silicate grains without having been sublimated (Mor \& Netzer 2012), thus calling for the presence of graphite grains in the galactic nuclei.

\section{Summary}

The AKARI all-sky survey and near- and far-IR spectroscopy have revealed various phenomena of processing of carbonaceous grains in harsh environments of galaxies. In particular, the $9 \mu \mathrm{m}$ diffuse map is the first all-sky map of the PAH emission. In this paper, based on spectroscopic observations, we present our results on two kinds of dust processing: structural changes of hydrocarbons and formation of graphite grains. From the galactic superwind of M82, the inner bar of NGC1097, and the foot points of the molecular loops near the Galactic center, we detect unusually strong aliphatic features at $3.4-3.6 \mu \mathrm{m}$ relative to the $3.3 \mu \mathrm{m}$ aromatic feature, which suggests shattering of carbonaceous grains by shocks. From the Galactic center, we detect a far-IR dust feature on top of a modified blackbody continuum around 110-130 $\mu \mathrm{m}$, which can be explained by micron-sized graphite grains. The detection of the dust feature from this region might be related to the (past) activity of the Galactic center, because the graphitization would suggest extreme thermal history. Similar situations are expected for AGN dusty tori and the centers of elliptical galaxies.

AKARI is a JAXA project with the participation of ESA. This research was supported by JSPS KAKENHI Grant Number 22340043, and the Nagoya University Global COE Program, "Quest for Fundamental Principles in the Universe: from Particles to the Solar System and the Cosmos", from MEXT.

\section{References}

Fukui, Y., et al. 2006, Science, 314, 106

Ishihara, D., et al. 2010, A\&A, 514, id.A1

Ishihara, D., Kaneda, H., Onaka, T., Ita, Y., Matsuura, M., \& Matsunaga, N. 2011, A\&A, 534, id.A79

Kaneda, H., Koo, B.-C., Onaka, T., \& Takahashi, H. 2009, Adv. Sp. Res., 44, 1038

Kaneda, H., et al. 2010, A\&A, 514, id.A14

Kaneda, H., et al. 2011, PASJ, 63, 601

Kaneda, H., et al. 2012a, PASJ, 64, 25

Kaneda, H., et al. 2012b, $A \mathscr{G} A$, 543, id.A79

Kawada, M., et al. 2007, PASJ, 59, S389

Kawada, M., et al. 2008, PASJ, 60, 389

Kondo, T., et al. 2012, ApJ, 751, L18

Kwok, S. \& Zhang, Y. 2011, Nature, 479, 80

Mor, R. \& Netzer, H. 2012, MNRAS, 420, 526

Murakami, H., et al. 2007, PASJ, 59, S369

Onaka, T., et al. 2007, PASJ, 59, S401

Yamagishi, M., et al. 2012, A\&A, 541, 10 